\title{
Using Square Wave Input for Wireless Power Transfer
}

\author{
Kazuya Yamaguchi*, Takuya Hirata*, and Ichijo Hodaka** \\ "Interdisciplinary Graduate School of Agriculture and Engineering, University of Miyazaki, Japan \\ *** Department of Environmental Robotics, Faculty of Engineering, University of Miyazaki, Japan
}

\section{Article Info}

\section{Article history:}

Received Sep 16, 2015

Revised Dec 19, 2015

Accepted Jan 2, 2016

\section{Keyword:}

Resonant phenomenon

Square wave

State space

Wireless power transfer

\begin{abstract}
A wireless power transfer (WPT) circuit is composed of a transmitting circuit with an AC power supply and a receiving circuit with a load, and the circuits are wirelessly connected each other. Then a designer chooses the wave form of the AC power supply. Many papers about WPT adopt a sinusoidal wave as the input. The frequency of the sinusoidal wave is generally determined to the resonant frequency of the circuit for high power transfer. Since the number of circuit elements in the power supply to generate a square wave is much less than that of a sinusoidal wave, WPT with a square wave input should be treated. In fact, some papers adopt a square wave as the input, and adjust the frequency of the square wave to the resonant frequency of the circuit. In this paper, we examine how the frequency of a square wave input affects power and efficiency of WPT circuits, and propose a procedure how to determine the frequency of the input to improve power and efficiency. Finally we discuss which wave should be adopted as an input and how the frequency of the input should be determined, regardless of whether resonant phenomena occur or not.
\end{abstract}

Copyright (c) 2016 Institute of Advanced Engineering and Science. All rights reserved.

\section{Corresponding Author:}

Ichijo Hodaka

Department of Environmental Robotics, Faculty of Engineering, University of Miyazaki

1-1, Gakuen Kibanadai Nishi, Miyazaki, 889-2192, Japan

Email: hijhodaka@cc.miyazaki-u.ac.jp

\section{INTRODUCTION}

In these years, technology of power transfer without electric wires between a power supply and a device to be powered, which is called wireless power transfer (WPT), has been actively studied. WPT is expected to make our life convenient at many situations. One is that the electric vehicles will be charged just by parking them at a specified place. Especially, many systems to charge the electric vehicles at the distance of dozens of centimeters with more than 80 percent efficiency are already reported by many developers of WPT. On the other hand, there is a problem that a power transfer is not suited for long distance since it is difficult to avoid the leakage inductances between a power supply and the devices.

Since an efficient power transfer was reported where the gap of transmission was 0.6 meters by [1], many studies about WPT have been reported. [2] presented a mathematical expression of efficiency with the quality factor $\mathrm{Q}$ instead of the circuit constants, and investigated how efficiency depended on Q. A mathematical expression of efficiency with Q for another WPT circuit is derived for series and parallel circuits in [3]. The expressions of efficiency and the optimal resistive load with a relay circuit between a transmitting and a receiving circuits are derived in terms of $\mathrm{Q}$ in [4], and it is shown that $\mathrm{Q}$ increases if a relay circuit is inserted between a transmitting circui and receiving circuit [5]. An equivalent circuit to WPT is used in order to analyze WPT circuits in [6]. The electromagnetic field with resonant phenomena is analyzed by [7]. It is proved that a DC power supply can be utilized as an input of WPT with an oscillator in [8].

Many papers about WPT adopt a sinusoidal wave as an input of a WPT circuit. [9] and [10] argue that a square wave is approximated as a sinusoidal wave which has the same frequency as the frequency of the square wave with resonant phenomena. This is attributed to the fact that if the fundamental frequency of the square wave which has many frequencies is adjusted to the resonant frequency, the influence of frequencies apart from the fundamental frequency will be suppressed. In fact, the range of frequency by a power supply does not always contain the resonant frequency. This situation should be investigated as well as driving the power supply at the resonant frequency. 
In this paper, we clarify how the driving frequency of a square wave input affects output power and efficiency of WPT, while many papers adopt a sinusoidal wave as an input. We calculate the values of output power and efficiency without approximating the square wave as a sinusoidal wave. The calculation is executed for three types of the circuits, and the difference between output powers with the sinusoidal and the square waves is examined in view of the characteristics of these waves and resonant phenomena. Finally, we discuss which waves should be adopted as an input on each situations, and decide the frequency of the periodic waves to realize a high power WPT.

\section{THE AMPLITUDES OF INPUT VOLTAGES}

A circuit which is commonly utilized for WPT is shown in Figure 1, and we consider the circuit for a simple WPT. $L_{1}, L_{2}$ are the self inductances to transfer energy electromagnetically, and $M$ is the mutual inductance which is determined by the radius, the number of turns, and the distance of the coils. The load $R_{\mathrm{L}}$ is resistive such as a battery. $R_{1}, R_{2}, C_{1}$, and $C_{2}$ are the parasitic factors of the transmitting circuit and receiving circuit.

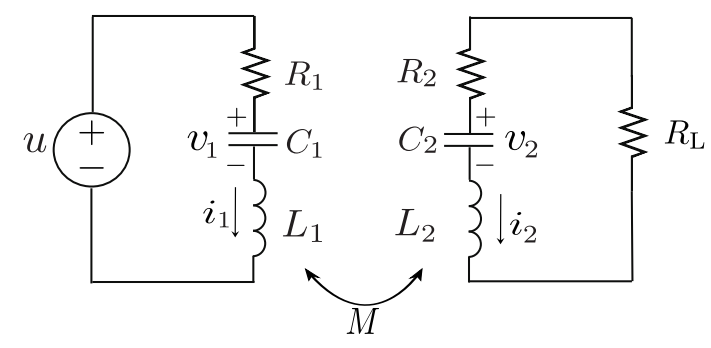

Figure 1. A simple WPT circuit

Here we assume that the input voltage $u$ is either a sinusoidal wave or a square wave, and we will investigate in the sequel how the choice of wave form improves or contaminates the system of WPT. In order to figure out results from the choice of input wave form, we should determine the amplitude of wave forms for the inputs to have the same output power. Then, we consider the bare circuit in Figure 2 instead of Figure 1. In the circuit in Figure 2, the power supply and the load (put $R_{\mathrm{L}}=100[\Omega]$ ) are borrowed from Figure 1. If we consider a sinusoidal wave and a square wave with amplitudes described in the table in Figure 2, we have the same output average power $5 \mathrm{~mW}$ in the table. Thus we adopt the amplitudes of voltages in the sequel of this paper.

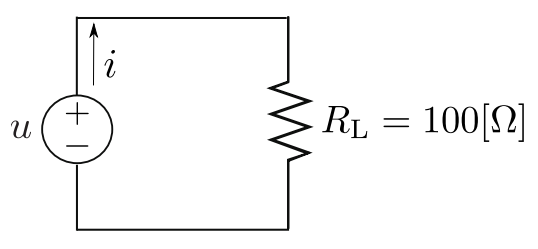

\begin{tabular}{|c|c|c|}
\hline & sinusoidal & square \\
\hline $\begin{array}{c}\text { voltage } \\
\text { with amplitude } 1 \mathrm{~V}\end{array}$ & $-v_{\overline{0}} \bar{V} I$ & $\uparrow 1 \mathrm{~V}$ \\
\hline $\begin{array}{c}\text { instantaneous } \\
\text { power } \\
u(t) \times i(t)\end{array}$ & 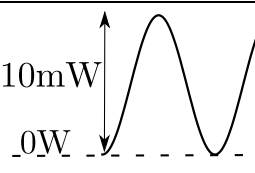 & $\begin{array}{l}\uparrow 10 \mathrm{~mW} \\
-0 \overline{\mathrm{W}}\end{array}$ \\
\hline $\begin{array}{c}\text { average } \\
\text { power } \\
\frac{1}{T} \int_{0}^{T} u(t) i(t) d t\end{array}$ & $5 \mathrm{~mW}$ & $5 \mathrm{~mW}$ \\
\hline
\end{tabular}

Figure 2. A bare circuit (left) and a table of voltage and input power (right)

\section{INPUT AND OUTPUT POWER, AND EFFICIENCY}

The behavior of WPT circuit in Figure 1 is governed by Kirchhoff's law, Ohm's law, and other laws of electric elements. Those laws can be described by algebraic and differential equations with time-variables of voltages and currents in the circuit. After eliminating unnecessary variables from the equations, we have differential equations driven by the input voltage as in the equation (1) that is called the state space equation, since the state variables $v_{1}, v_{2}$, 
$i_{1}$ and $i_{2}$ represent the whole behavior of the circuit. If we choose the current $i_{2}$ as an output of the system, we can express the input to output behavior as a transfer function written by (2).

$$
\begin{aligned}
\dot{x} & =A x+B u, x=\left[\begin{array}{llll}
v_{1} & v_{2} & i_{1} & i_{2}
\end{array}\right]^{\mathrm{T}} \\
A & =\frac{1}{\Delta}\left[\begin{array}{cccc}
0 & 0 & \frac{\Delta}{C_{1}} & 0 \\
0 & 0 & 0 & \frac{\Delta}{C_{2}} \\
-L_{2} & M & -R_{1} L_{2} & R_{3} M \\
M & -L_{1} & R_{1} M & -R_{3} L_{1}
\end{array}\right], B=\frac{1}{\Delta}\left[\begin{array}{c}
0 \\
0 \\
L_{2} \\
-M
\end{array}\right] \\
\Delta & =L_{1} L_{2}-M^{2}, R_{3}=R_{2}+R_{\mathrm{L}}
\end{aligned}
$$

$$
G(s)=\frac{-M C_{1} C_{2} s^{3}}{\Delta C_{1} C_{2} s^{4}+\left(R_{1} L_{2}+R_{3} L_{1}\right) C_{1} C_{2} s^{3}+\left(L_{1} C_{1}+L_{2} C_{2}+R_{1} R_{3} C_{1} C_{2}\right) s^{2}+\left(R_{1} C_{1}+R_{3} C_{2}\right) s+1}
$$

In order to visualize the input to output relation, we substitute concrete values into values of circuit elements as in the table below. Then we have three log-log plots respectively for the sinusoidal input (left column) and the square wave input (right column) in Figure 3. The first row of plots is the gain plots of transfer function $G(s)$ in (2). The second row depicts the average power $P_{1}$ at the power supply and $P_{2}$ at the load. The third row is the efficiency which is defined by the ratio of the output power $P_{2}$ to the input power $P_{1}$. The horizontal axes are all the angular frequency of input voltages. Needless to say, the ouput power $P_{2}$ and efficiency are desired to be higher for any WPT circuit. A designer or user of WPT circuits choose the best driving frequency in view of the last two rows of plots. 

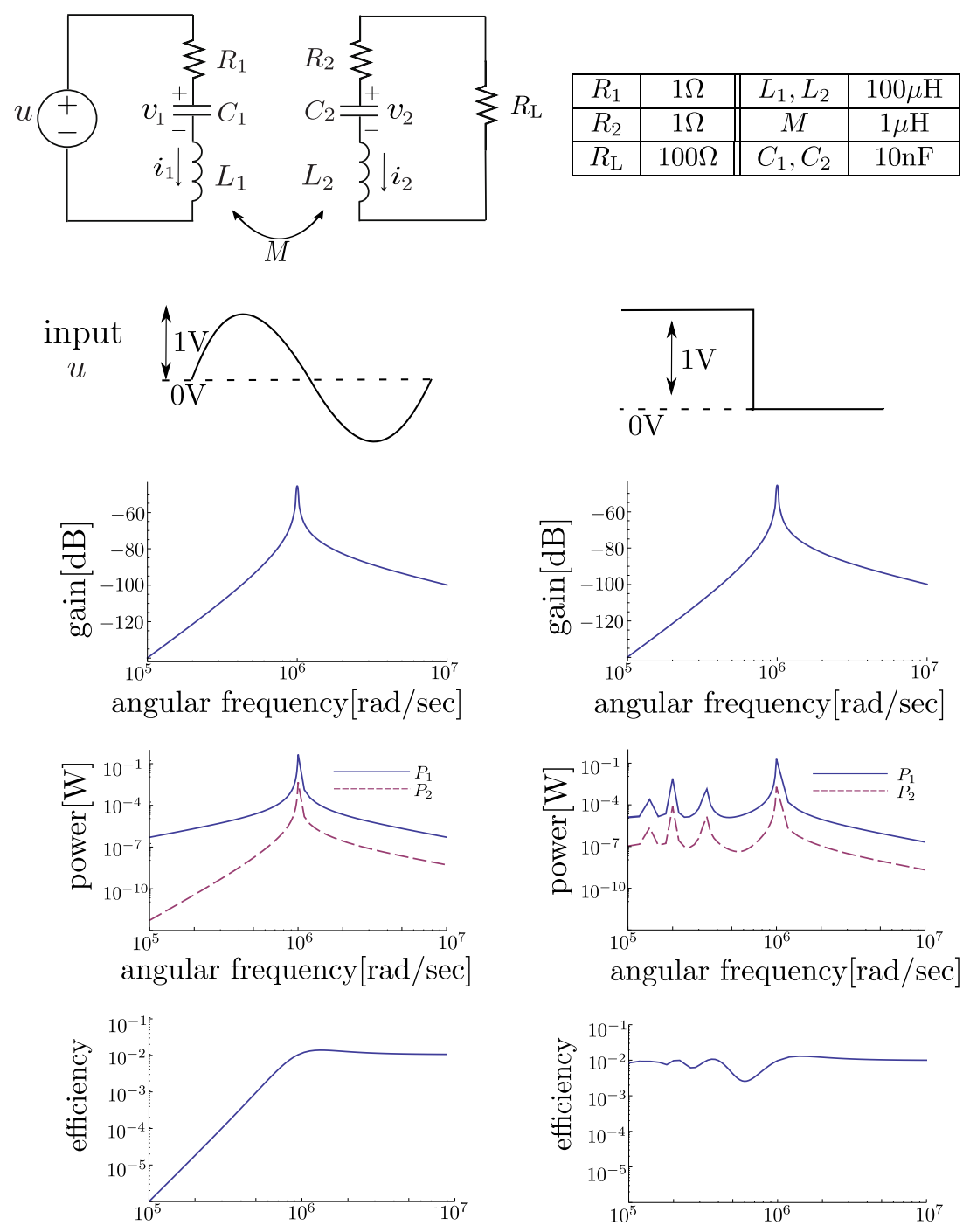

angular frequency $[\mathrm{rad} / \mathrm{sec}]$

Figure 3. Gain, power, and efficiency versus frequency

Notice that the resonant angular frequency is equal to $10^{6} \mathrm{rad} / \mathrm{sec}$, as seen in the first row (two plots in the first are identical since the transfer function is uniquely determined by the circuit; it does not depend on what input wave is used). In this case, the peak frequency in power is equal to that in gain, although that is not always true in general. Moreover, the efficiency reaches maximum at the peak frequency. This is the case in both of sinusoidal wave and square wave inputs. The difference of left and right plots is the number of local peaks in power and efficiency. This comes, of course, from that square waves have many different frequencies, and that voltages and currents are given by superposition of ones by separate sinusoidal inputs with different frequencies. In the following section, we will discuss the situation above in a mathematical way.

\section{CALCULATION OF POWER BY SQUARE WAVE INPUT}

The partial sum of Fourier series of a square wave with the amplitude 1 is written by the following.

$$
\widetilde{u_{\mathrm{sq}}}=\frac{1}{2}+\frac{2}{\pi} \sum_{k=1}^{n} \frac{1}{2 k-1} \sin (2 k-1) \omega t
$$

where $\omega=2 \pi / T$ and $T$ is the period of the square wave. If we put $n \rightarrow \infty$, the above converges the square wave pointwizely except for the uncontinuous points. Since the WPT circuit consists of linear and time-invariant elements, the state space representation is also linear and time-invariant as in (1). Then we have transfer functions from the 
input $u$ to any of state variables $v_{1}, v_{2}, i_{1}$ and $i_{2}$. Therefore, it is straightforward to obtain the periodic and stationary solution to the equation (1) with the periodic input (3). If we put the number $n$ in (3) as large enough, we have approximated solutions to the exact solutions with square wave inputs.

Here note that we should focus on power which is product of voltage and current, when we refer to the performance of WPT system. Since the relation between input voltage and power at the load is not linear, it is not straightforward to obtain the mathematical expression of power. Nevertheless, we can deduce a simple expression of power. For this end, we write $P(u)$ as the average power at the load by a periodic input $u$. It can be verified that $P\left(u_{1}+u_{2}\right)=P\left(u_{1}\right)+P\left(u_{2}\right)$ holds if $u_{1}$ and $u_{2}$ are orthogonal, that is, $\int_{0}^{T} u_{1}(t) u_{2}(t) d t=0$, where $T$ is the period of $u_{1}$ and $u_{2}$. Therefore $P\left(\widetilde{u_{\mathrm{sq}}}\right)$ is written as

$$
P\left(\widetilde{u_{\mathrm{sq}}}\right)=P\left(\frac{1}{2}\right)+P\left(\frac{2}{\pi} \sin \omega t\right)+P\left(\frac{2}{3 \pi} \sin 3 \omega t\right)+\cdots+P\left(\frac{2}{(2 n-1) \pi} \sin (2 n-1) \omega t\right) .
$$

Another apparant but important identity is $P(\alpha u)=\alpha^{2} P(u)$ ( $\alpha$ is an arbitrary real number). Then $P\left(\widetilde{u_{\mathrm{sq}}}\right)$ in $(4)$ is refined as

$$
P\left(\widetilde{u_{\mathrm{sq}}}\right)=\left(\frac{2}{\pi}\right)^{2} \sum_{k=1}^{n}\left(\frac{1}{2 k-1}\right)^{2} P(\sin (2 k-1) \omega t)
$$

The equation (5) gives how to calculate the power with square wave inputs based on power with sinusoidal wave inputs. A rough estimation for (5) is given by

$$
P\left(\widetilde{u_{\mathrm{sq}}}\right)=0.41 P(\sin \omega t)+0.045 P(\sin 3 \omega t)+0.016 P(\sin 5 \omega t) .
$$

The coefficients $0.41,0.045$ and 0.016 respectively reflect how the powers $P(\sin \omega t), P(\sin 3 \omega t)$ and $P(\sin 5 \omega t)$ with sinusoidal inputs respectively contribute to the power $P()$ with the square input. With this observation, it is reasonable that we should adjust the driving frequency of square wave input to the resonant frequency of WPT circuit, just like sinusoidal wave input, when we intend to obtain high output power. This could be true if the power plot versus frequency with sinusoidal inputs has a single steep peak. In other words, we may neglect higher frequencies and concentrate on the fundamental frequency, also stated in $[9,10]$.

For some WPT circuits, power plot versus frequency with sinusoidal inputs inevitably have plural or gentle peaks, where we should take higher frequencies as well as the fundamental frequency into account. One important observation is to see a local peak at one third frequency of the resonant frequency as the power plot with square wave inputs in Figure 3, because square wave has three times frequency of the fundamental frequency. Such situations are, in general, difficult to cope with, and little is known, or even not referred to in the literature.

Plot of efficiency is not always monotone in general, even if gain plot and power plot are monotone as in Figure 3. In fact, maximizing output power does not always mean maximizing efficiency as discussed in [11, 12, 13].

\section{POWER AND EFFICIENCY WITH THE VARIOUS CIRCUITS}

Power and efficiency about the circuits of Figure 4 and Figure 5 are shown below. A WPT circuit with a relay circuit in Figure 5 can be efficient in order to increase a distance between a power supply and a resistive load to transfer power[14]. Here we omit to write the state space representation and transfer function, and readers can refer to [15] about the calculation and expression of them.

These results show that the average powers with square wave inputs are locally high at the neighborhoods of the resonant frequency and one third of the resonant frequency as in Figure 3. As we can see these results, if the frequency of input is restricted to values lower than the resonant frequency, we should use a square wave rather than a sinusoidal wave as a voltage input of WPT circuit. 


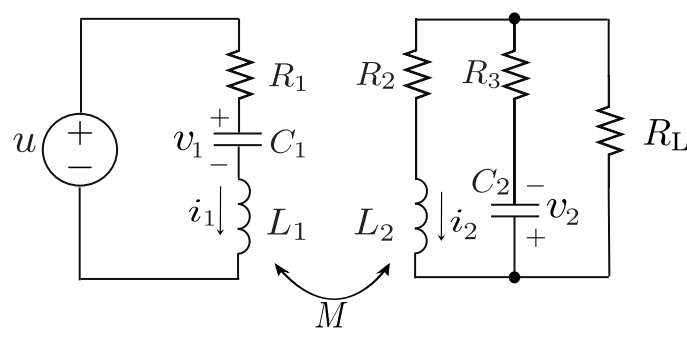

\begin{tabular}{|c|c||c|c|}
\hline$R_{1}$ & $1 \Omega$ & $L_{1}, L_{2}$ & $100 \mu \mathrm{H}$ \\
\hline$R_{2}$ & $1 \Omega$ & $M$ & $1 \mu \mathrm{H}$ \\
\hline$R_{3}$ & $1 \Omega$ & $C_{1}, C_{2}$ & $10 \mathrm{nF}$ \\
\hline$R_{\mathrm{L}}$ & $100 \Omega$ & & \\
\hline
\end{tabular}
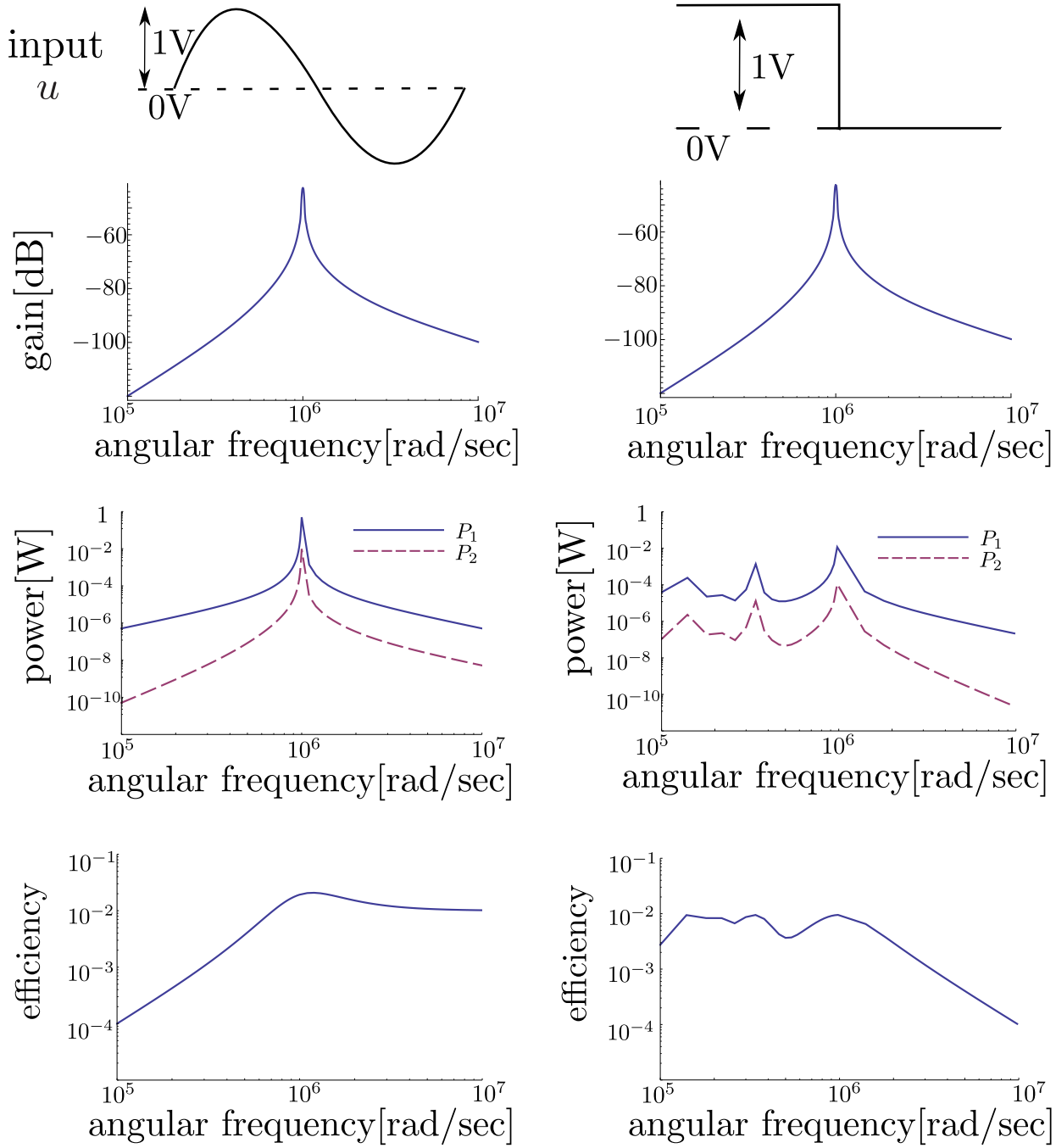

Figure 4. Bode diagram and power and efficiency with a parallel circuit 


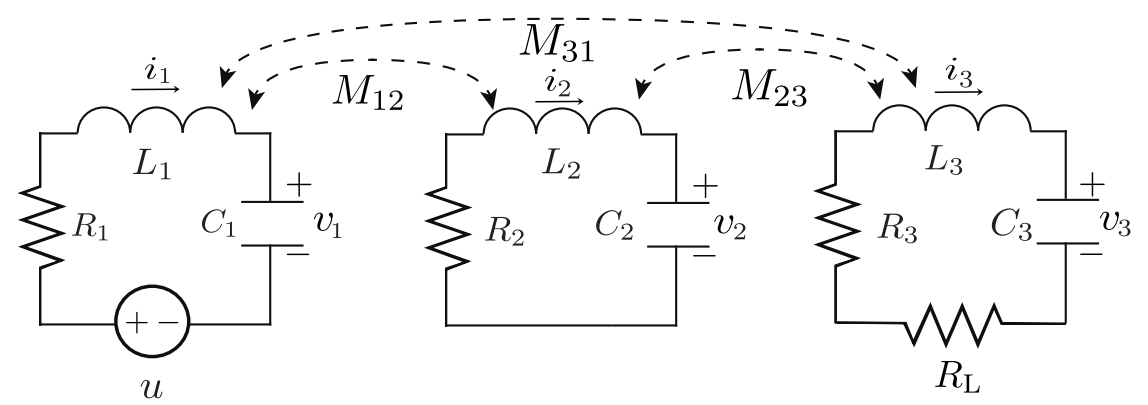

\begin{tabular}{|c|c||c|c|}
\hline$R_{1}, R_{2}$ & $1 \Omega$ & $L_{1}, L_{2}, L_{3}$ & $100 \mu \mathrm{H}$ \\
\hline$R_{3}$ & $0.1 \Omega$ & $M_{12}, M_{23}$ & $10 \mu \mathrm{H}$ \\
\hline$R_{\mathrm{L}}$ & $100 \Omega$ & $M_{31}$ & $1 \mu \mathrm{H}$ \\
\hline$C_{1}, C_{2}, C_{3}$ & $10 \mathrm{nF}$ & & \\
\hline
\end{tabular}
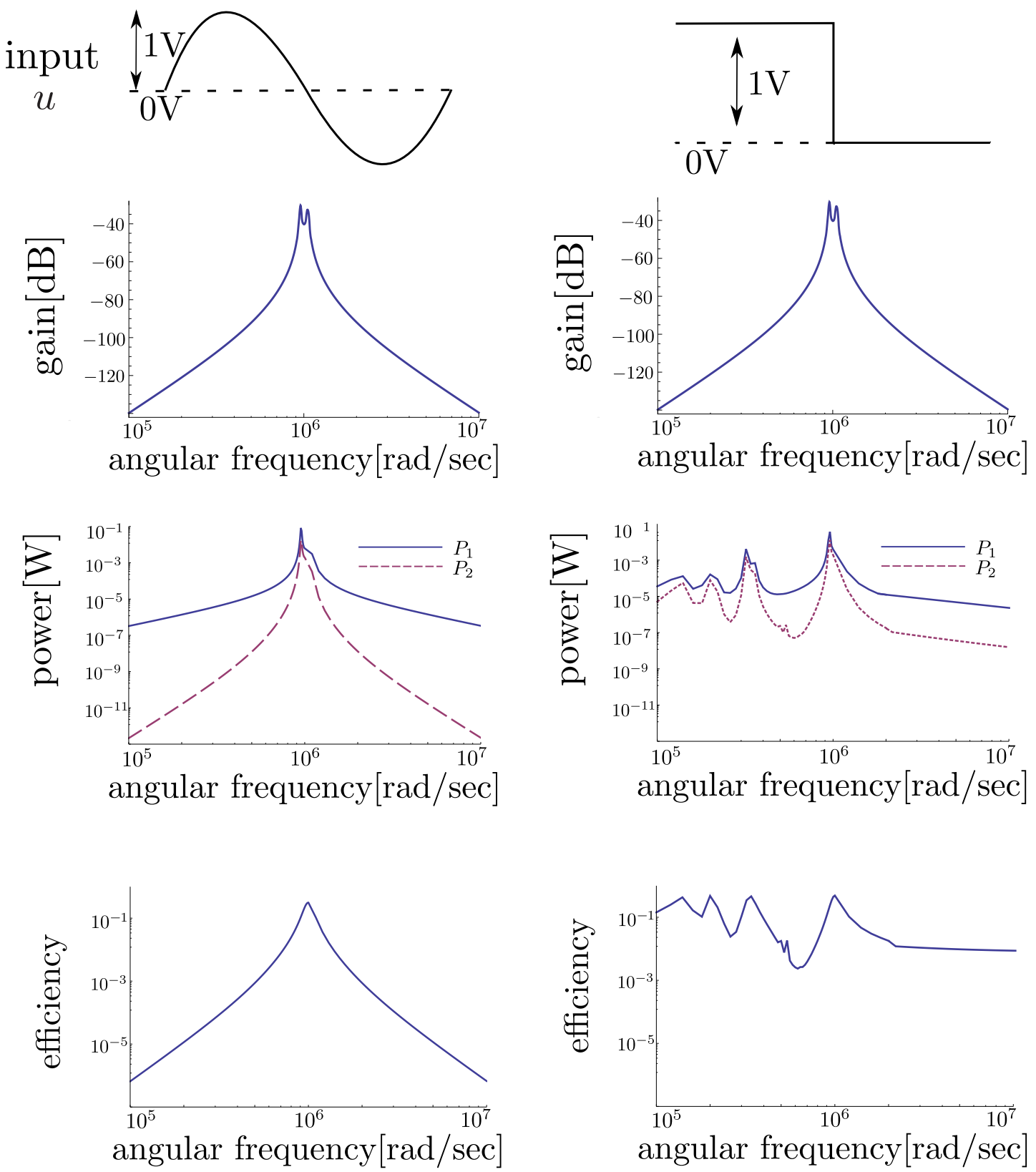

Figure 5. Bode diagram and power and efficiency with a relay circuit 


\section{CONCLUSION}

In this paper, we have examined how power and efficiency of wireless power transfer are affected by choosing type of wave form of AC power supply voltage, sinusoidal waves and square waves, and then, we have suggested which type of wave form should be chosen for a better wireless power transfer. We have confirmed that the frequency of AC power supply should be adjusted to the resonant frequency of the circuit if the sinusoidal input is chosen, as many other papers also concluded. However, it is not always possible to use the resonant frequency, especially if the frequency is too high to implement the synchronous input. For such a difficult situation, we have proposed to utilize the characteristics that square waves contain (infinitely) many frequencies. Then we have shown that the higher power can be obtained even if the frequency of input is restricted to a lower frequency than the resonant frequency. This gives a new insight for the development of wireless power transfer system driven by square wave inputs.

\section{REFERENCES}

[1] A. Kurs, A. Karalis, R. Moffatt, J. D. Joannopoulos, P. Fisher, M. Soljačić. Wireless Power Transfer via Strongly Coupled Magnetic Resonances. Science. 2007; 317: 83-86.

[2] C. A. Tucker, K. Warwick, W. Holderbaum. A contribution to the wireless transmission of power. Electrical Power and Energy Systems. 2013; 47: 235-242.

[3] G. Vandevoorde, R. Puers. Wireless energy transfer for stand-alone systems: a comparison between low and high power applicability. Sensors and Actuators A: Physical. 2001; 92: 305-311.

[4] T. Takura, T. Misawa, F. Sato, T. Sato, H. Matsuki. Maximum Transmission Efficiency of LC-Booster Using Pick-up Coil with Capacitance. Journal of the Magnetics Society of Japan. 2013; 37: 102-106 (in Japanese).

[5] H. Hoang, F. Bien. Maximizing Efficiency of Electromagnetic Resonance Wireless Power Transmission Systems with Adaptive Circuits. Wireless Power Transfer - Principles and Engineering Explorations. 2012; 207-226.

[6] T. Imura, Y. Hori. Maximizing Air Gap and Efficiency of Magnetic Resonant Coupling for Wireless Power Transfer Using Equivalent Circuit and Neumann Formula. IEEE Transactions on Industrial Electronics. 2011; 58: 4746-4752.

[7] L. L. Tan, X. L. Huang, H. Huang, Y. W. Zou, H. Li. Transfer efficiency optimal control of magnetic resonance coupled system of wireless power transfer based on frequency control. Science China Technological Sciences. 2011; 54: 1428-1434.

[8] A. N. Laskovski, M. R. Yuce. Class-E self-oscillation for the transmission of wireless power to implants. Sensors and Actuators A: Physical. 2011; 171: 391-397.

[9] K. Hata, T. Imura, Y. Hori. Maximum Efficiency Control of Wireless Power Transfer Using Secondary Side DCDC Converter for Moving EV in Long Distance Transmission. Technical Report of IEICE. 2014; 114: 51 -56 (in Japanese).

[10] J. de Boeij, E. Lomonova, J. L. Duarte, A. J. A. Vandenput. Contactless power supply for moving sensors and actuators in high-precision mechatronic systems with long-stroke power transfer capability in x-y plane. Sensors and Actuators A: Physical. 2008; 148: 319-328.

[11] K. Yamaguchi, T. Hirata, Y. Yamamoto, I. Hodaka. Resonance and efficiency in wireless power transfer system. WSEAS Transactions on Circuits and Systems. 2014; 13: 218-223.

[12] S. G. Lee, H. Hoang, Y. H. Choi, F. Bien. Efficiency improvement for magnetic resonance based wireless power transfer with axial-misalignment. Electronics Letters. 2012; 48: 339-340.

[13] K. Yamaguchi, Y. Yamamoto, T. Hirata, E. Setiawan, I. Hodaka. Mathematical Expression of Optimal Frequencies for Wireless Power Transfer. Proceedings of The 3rd International Conference on Computer Engineering \& Mathematical Sciences. 2014; 826-827.

[14] D. Niu, K. Shuang, W. Li. Magnetic Resonant Coupling for Magnetic Induction Wireless Communication. IETE Journal of Research. 2013; 59: 624-630.

[15] T. Hirata, Y. Yamamoto, K. Yamaguchi, E. Setiawan, I. Hodaka. On Circuit Topologies of Wireless Power Transmission with Relay Coils. Proceedings of The 3rd International Conference on Computer Engineering \& Mathematical Sciences. 2014; 200-202. 